\title{
Apuntes Conceptuales sobre Sostenibilidad y Responsabilidad Social Empresarial en el Contexto Latinoamericano
}

\author{
Conceptual Notes on Sustainability and Corporate Social \\ Responsibility in the Latin American Context
}

\begin{abstract}
RESUMEN
Este artículo pretende contribuir al debate sobre la Responsabilidad Social Empresarial desde el contexto latinoamericano, el cual exige la inclusión de elementos que no son suficientemente abordados en la literatura anglosajona y cuya relevancia es inferior en comparación a lo que puede ocurrir en áreas distintas a las que corresponden a países con mayor nivel de desarrollo. En ese sentido, se presentan algunos elementos clave en la caracterización de un contexto particular como el latinoamericano, como es el caso de la corrupción, para entender la naturaleza de las vinculaciones entre empresa y sociedad, sus efectos y motivaciones. En la revisión de la literatura se encontraron diversos conceptos cuya definición resultó ambigua y que pueden provocar inexactitudes teóricas que no ayudan a la descripción de los fenómenos sociales. Asimismo, el criterio para seleccionar los conceptos discutidos en este artículo ha sido el de la instrumentalización; es decir, resaltar aquellos conceptos que faciliten la conexión entre elementos de naturaleza ética y económica. Finalmente, se propuso una tipología para describir las vinculaciones entre empresa y sociedad desde la Responsabilidad Social Empresarial.
\end{abstract}

Palabras claves: responsabilidad social empresarial; grupos de interés; sostenibilidad empresarial.

\begin{abstract}
This article aims to contribute to the discussion on Corporate Social Responsibility from the Latin American context, which requires the inclusion of elements that are not adequately addressed in the American and European literature and whose relevance is lower compared to what happen in geographical areas of countries with a higher level of development. In that sense, some key elements are presented in the characterization of a particular context such as Latin American, as is the case of corruption, to understand the nature of the links between business and society, its effects and motivations. In the literature review, several concepts were found whose definition was ambiguous and which can cause theoretical inaccuracies that do not help the description of social phenomena. Likewise, the criterion for selecting the concepts has been that of instrumentalization; that is, highlight those concepts that facilitate the connection between elements of an ethical and economic nature. Finally, a typology was proposed to describe the links between business and society.

Keywords: corporate social responsibility; stakeholders; corporate sustainability.
\end{abstract}

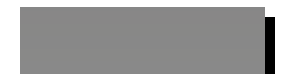

Bruno Chaihuaque Dueñas $^{1}$

bchaihuaque@pucp.pe

Pontificia Universidad Católica del Perú

\footnotetext{
${ }^{1}$ Departamento Académico de Ciencias de la Gestión, Pontificia Universidad Católica del Perú

() Los autores. Este artículo es publicado por Gestión en el Tercer Milenio de la Facultad de Ciencias Administrativas de la Universidad Nacional Mayor de San Marcos. Este es un artículo de acceso abierto, distribuido bajo los términos de la licencia Creative Commons Atribucion - No Comercia_Compartir Igual 4.0 Internacional. (http://creativecommons.org/licenses/by-nc-sa/4.0/) que permite el uso no comercial, distribución y reproducción en cualquier medio, siempre que la obra original sea debidamente citada.
} 


\section{INTRODUCCIÓN}

La Teoría de la Responsabilidad Social Empresarial ha experimentado una evolución interesante, habiéndose generado una serie de bifurcaciones conceptuales que intentan explicar distintos fenómenos producidos a partir de la relación empresa-sociedad. Se suele reconocer a Bowen (1953) como el iniciador del debate respecto a lo que debe entenderse como Responsabilidades Sociales de los Hombres de $\mathrm{Ne}$ gocios, y su posterior evolución a las múltiples concepciones, teorías, aproximaciones y terminologías referidas a la Responsabilidad Social Empresarial (Garriga \& Melé, 2004). Así pues, la necesidad de comprender la vinculación de la empresa con el entorno social ha generado una serie de conceptos que han sido concebidos desde una perspectiva relacionada con contextos occidentales, principalmente de Norteamérica.

La filantropía empresarial, la política social corporativa, gestión de grupos de interés, entre otros son aproximaciones que fueron concebidas a partir de los fenómenos suscitados en EE.UU. y países de Europa. Incluso algunos de estos conceptos y teorías son introducidos en textos y cursos de Responsabilidad Social o Ética Empresarial, los que en muchos casos ayudan a comprender fenómenos dentro de su ámbito de estudio. Sin embargo, el contexto Latinoamericano mantiene ciertas particularidades que es posible abordarlas mediante el desarrollo de concepciones diferentes y proposiciones teóricas distintas que incluyan aspectos propios de la realidad de los países de la región.

Uno de estos elementos es la corrupción, $\mathrm{y}$ aquellas acciones ejecutadas desde organizaciones que impactan negativamente en la sociedad. Dentro de lo que corresponde a los Estudios Organizacionales (Clegg \& Hardy, 1996), este fenómeno puede abordarse desde tres perspectivas distintas: (a) El Comportamiento Organizacional, particularmente la Ética en los Negocios; (b) la Ecología Organizacional, principalmente desde la perspectiva de la Teoría de los Grupos de Interés; y (c) la Teoría Organizacional, específicamente desde las Teorías de los Sistemas Organizacionales, la Nueva Teoría Institucional y la Teoría de Contingencia.

Como se ha dicho, el fenómeno de la corrupción y sus efectos en las organizaciones puede ser abordada desde diversos campos de los Estudios Organizaciones. Sin embargo, una búsqueda de los términos "Corruption" AND "Corporate Social Responsibility" arrojó 120 resultados para la base de datos SCOPUS (Con resultados limitados al campo "Business Management and Accounting”). Un ejercicio similar, esta vez realizado en la base de datos Web of Science arrojó 129 artículos vinculados con estas temáticas, para los sub campos "Business", "Management" y "Ethics". Es importante resaltar, que la mayor parte de resultados se presentan a partir del año 2008, coincidentemente el año en el que se ocurrieron los mayores efectos de la Crisis Financiera Internacional 2007-2008, como el colapso del Banco de Inversión Lehman Brothers y el inicio de la Crisis de Deuda Soberana Europea, hechos que han sido atribuidos entre otras causas al problema de Riesgo Moral oportunismo y asimetría de la información (Hnyilicza, 2009 \& Guillén, 2009).

Episodios de corrupción han producido efectos de enorme impacto para la sociedad (crisis financiera, pérdida de empleo y deterioro de la capacidad adquisitiva, entre otros), pero además efectos importantes para las empresas que incluso comprometen su sostenibilidad. Es por ello que se considera a la Responsabilidad Social Empresarial (RSE) como un concepto que no puede analizarse de manera aislada, sino que necesita ser abordado desde perspectivas más amplias para comprender con mayor prolijidad su naturaleza, en un contexto latinoamericano caracterizado por el escaso desarrollo de los mercados, informalidad y corrupción.

El presente artículo aborda el fenómeno de la vinculación de intereses en la relación empresa-sociedad, en base a los Grupos de Interés (GI) y la Sostenibilidad Empresarial (SE), sugiriendo la necesidad de incorporar una mayor precisión sobre los términos que se han venido utilizando en la formulación teórica. En ese sentido, se reafirma lo señalado por Freeman, Harrison, Wicks, Parmar \& de Colle (2010), respecto a la necesidad de generar una nueva narrativa respecto al rol de los tomadores de decisión en las empresas, en contraposición a las propuestas irreales sobre la confrontación entre el interés económico y el valor social. Por ese motivo, se considera indispensable utilizar 
los términos adecuados en la caracterización de los fenómenos y, a partir de estas definiciones, formular las refinaciones que correspondan a las propuestas teóricas relacionadas con la RSE.

\section{OBJETIVOS DEL ENSAYO}

El objetivo de este artículo es identificar aquellas definiciones que permitirían una mayor precisión en la formulación teórica de fenómenos vinculados con la corrupción desde la Teoría de la RSE en el contexto Latinoamericano. En ese sentido, se discute sobre la definición de los conceptos de GI y SE. Más aun, se analiza el concepto de SE, debido a las distintas acepciones que podrían dificultar el debate entre la academia y la empresa. En virtud a ello, se expone un análisis de los conceptos planteados en la Teoría de la RSE y SE, desde los planteamientos medioambientales, hasta los que se vinculan con la capacidad para la generación de rentas en el largo plazo. Finalmente, el artículo incluye una propuesta de clasificación de las relaciones entre empresa y sociedad, desde la RSE.

\section{ARGUMENTACIÓN}

\section{Las múltiples acepciones de sostenibili- dad empresarial.}

La SE es un constructo con múltiples definiciones. El origen del concepto tiene una connotación medioambiental. Precisamente, Hans Carl von Carlowitz expone en su libro "Sylvicultura oeconomica, oder haußwirthliche Nachricht und Naturmäßige Anweisung zur wilden Baum-Zucht" (Silvicultura Económica o las Instrucciones para el Cultivo de Árboles Salvajes) ideas sobre el uso sostenible de los bosques y una ética del manejo responsable de los recursos naturales. Estas ideas dieron origen a la noción de umbral de resultados (McElroy \& Van Engelen, 2012).

En años recientes, la SE ha ocupado un espacio importante en la agenda social, en virtud al reconocimiento de que la actividad humana puede alterar las condiciones sobre las cuales se regeneran los recursos. Ello ha sido motivo de alerta en diversos foros. Así pues, en 1972 las Naciones Unidas (NU) organizó la Conferencia sobre el Medio Ambiente Humano, en donde se expuso la necesidad de formular políticas que inspiren a los gobiernos y organizaciones a tomar acción sobre el cuidado y preservación del medioambiente bajo una mirada de sostenibilidad.

Sucesivos eventos y foros organizados por las NU han reforzado esta acepción de Sostenibilidad. Por ejemplo, el establecimiento del Protocolo de Kioto en 1997, el Pacto Mundial del año 2000 o los Objetivos de Desarrollo Sostenible. Este último ligado a la lucha contra la corrupción y una mayor conciencia sobre los efectos permisivos de ésta en la sociedad (United Nations Global Compact, 2018).

En ese sentido, las NU Global Compact se establece como instrumento para guiar las acciones de organizaciones privadas, principalmente desde una perspectiva social, ambiental y de desarrollo sostenible. Otra acepción al concepto de sostenibilidad está referida a la capacidad de la empresa para mantener sus operaciones en el largo plazo, a partir de la generación de flujos económicos que sean suficientes para cubrir los costos, gastos económicos y financieros y que además permitan la creación de valor privado. En esta línea se ubican los conceptos expuestos por Dyllick \& Hockerts (2002), Aras \& Crowther (2009), Bansal \& Desjardine (2014), entre otros.

En contraste, el concepto de Triple Resultado, propuesto por Elkington (1994), trata de agregar las perspectivas ecológicas, sociales y económicas, en lo que se conoce como el modelo de las 3P (people, planet and profits). Los planteamientos de Elkington han motivado el interés por formular propuestas que incluyan los tres elementos descritos. En ese orden de ideas, podemos encontrar los artículos de Van Marrewijk (2003), Bansal (2005), Hahn (2011), Montiel \& Delgado-Ceballos (2014). Precisamente Montiel \& Delgado-Ceballos proponen que el constructo SE debe contener los tres pilares propuestos en la Teoría del Triple Resultado: Economía, Sociedad y Medioambiente que permiten un alineamiento de diez dimensiones: (a) crecimiento económico, (b) valor para los accionistas, (c) prestigio, (d) reputación empresarial, (e) relaciones con el cliente, (f) calidad de producto, (g) ética en los negocios, (h) creación de fuentes de empleo sostenible, (i) creación de valor para todos los GI y (j) atención de las necesidades de los desatendidos. La SE se presenta como un 
instrumento para la consecución de objetivos internos e inherentes de la empresa, como la creación de valor económico, y objetivos externos vinculados a la creación de valor social.

\section{La legitimidad de intereses en la identi- ficación de los grupos de interés.}

Uno de los hitos más importantes en la evolución del concepto de RSE es la crítica que Friedman (13 de Setiembre de 1970:1) realiza precisamente al concepto de Responsabilidad Social. Friedman señala en este artículo lo siguiente:

The discussions of the "social responsibilities of business" are notable for their analytical looseness and lack of rigor. What does it mean to say that "business" has responsibilities? Only people have responsibilities. A corporation is an artificial person and in this sense may have artificial responsibilities, but "business" as a whole cannot be said to have responsibilities, even in this vague sense.

La idea central que expone Friedman es el conflicto de interés que se produce en los gerentes y directores de las empresas que ejecutan acciones de responsabilidad social frente a los intereses de los accionistas y sus expectativas de creación y captura de valor privado. En ese sentido, este autor expone argumentos que sustentan su posición respecto a la prevalencia y exclusividad del interés de los inversionistas, sobre cualquier motivación que suponga una acción de responsabilidad social y que afecte estos intereses en la medida que se destine parte del valor privado creado por la empresa. Lo interesante del planteamiento de Friedman es que pone a la luz una idea que, aun siendo polémica, podría representar el sentir de los accionistas. En todo caso, releva la importancia de la responsabilidad fiduciaria de la gerencia respecto al interés de los accionistas.

La Teoría de los Grupos de Interés y su consolidación a partir de las ideas expuestas por Freeman (1984) trajo a debate la naturaleza multi fiduciaria de la responsabilidad empresarial. Freeman señala que para que las empresas sean exitosas en el largo plazo (sostenibles), los gerentes deben tener una respuesta precisa a la pregunta ¿a quienes representamos? (1984). La Teoría de los Grupos de Interés coloca en la agenda de los tomadores de decisión y quienes formulan las estrategias empresariales la necesidad de expandir la función de la gerencia para acoger los intereses, no necesariamente económicos, de los grupos alrededor de la empresa, atendiendo consideraciones legales y morales, y sin comprometer el futuro de la misma empresa, para que se formulen acciones que atiendan a estos agentes.

Una precisión importante se desprende de las ideas expuestas por Donaldson \& Preston (1995), quienes señalan que los GI son personas o grupos con legítimos intereses sobre aspectos sustantivos o procedimentales de la actividad de una empresa en particular. Se excluye a aquellos agentes cuyos intereses no son legítimos, moral y éticamente correctos e incluso ilegales. Esta definición es importante porque refuerza la conexión entre las Teorías de Grupos de Interés y RSE. En ese orden de ideas, cabe preguntarse si el interés de un agente es suficiente para aceptar sus reclamaciones. La hipótesis de Donaldson \& Preston (1995) excluye la posibilidad de integración de agentes con intereses ilegítimos dentro de los GI, y con ello acota la RSE.

Por lo tanto, se propone que aun cuando agentes con intereses ilegítimos formen parte del entorno en el que operen las empresas, su presencia puede ejercer presiones que se presentan de manera contingente, impactando en la empresa (y viceversa), pero no son parte de los GI sobre los cuales las empresas tengan responsabilidad para su atención. Sin embargo, su presencia merece un abordaje distinto para la caracterización del entorno. Concretamente, el fenómeno de la corrupción tiene una naturaleza diversa, debido a que su presencia, manifestada en la dinámica de intereses ilegítimos, está vinculada con inercia y cambio organizacional, mortalidad organizacional, isomorfismo institucional y estructuras que trascienden la organización (Teoría de Redes). Algunas publicaciones que han contribuido al debate del fenómeno de la corrupción desde distintas perspectivas de los Estudios Organizacionales son las de Michael (2004); Venard (2009); Jacobs, Van Witteloostuijn \& Christe-Zeyse (2013); Martin, Johnson \& Cullen (2015); entre otros.

Asimismo, en diversas publicaciones se ha explorado la relación entre corrupción y destrucción de valor social. Al respecto, Aidt 
(2011) explica que la corrupción impacta en el desarrollo sostenible. Más aun, recientes debates sobre la responsabilidad de las empresas en las crisis financieras han motivado la irrupción de conceptos novedosos como el de la Irresponsabilidad Social Empresarial (ISE). Este concepto surge desde la observación formulada a la RSE, su ambigüedad funcional y su escasa contribución en la institucionalización de prácticas relacionadas con la ética empresarial. Tench, Sun \& Jones (2012) señalan que el fracaso de la RSE debe ser analizado desde su concepción teórica hasta su puesta en práctica. Así pues, estos autores resaltan las contradicciones presentadas desde la concepción teórica de los modelos de los GI y de la creación de valor privado para los accionistas. Estos modelos que constituyen la piedra angular de la RSE mantienen objetivos divergentes. En un caso se trata de negocios sin ética y en el otro pilar se tiene ética sin negocio. En cuanto a la práctica, la ambigüedad en la definición de la RSE propicia la discrecionalidad en la ejecución de acciones que, siendo legales, no necesariamente son éticamente correctas para la sociedad. La RSE no representa un lindero claro para la separación de lo moral y lo legal. En consecuencia, los ejecutores de la acción empresarial se ven tentados a formular y elegir acciones cuyo impacto social es escaso e incluso negativo, pero con un mayor impacto en lo privado.

En virtud a lo expuesto, las vinculaciones entre empresa y sociedad se producen en tres escenarios. El primero de ellos ocurre cuando la empresa se vincula con GI. En este caso, la vinculación proviene de la reclamación del grupo de interés y se refuerza en la medida que haya una correspondencia en la empresa. La acción empresarial supone el incremento y captura de valor económico sin afectar la creación de valor social (inclusión). El segundo escenario corresponde a la vinculación de la empresa con agentes con los que comparten intereses, siendo estos ilegítimos. Este escenario supone la coordinación entre la empresa y estos agentes para la captura y reparto de valor privado en desmedro del valor social (colusión). El tercer escenario no supone la vinculación de la empresa con otros agentes, sino el abuso mediante la posición de dominio en la relación empresa-grupo de interés. En este caso, la motivación es la captura de valor económico en desmedro del valor social (dominación). La Figura 1 representa las tipologías definidas en estos tres escenarios de inclusión, colusión y dominación.

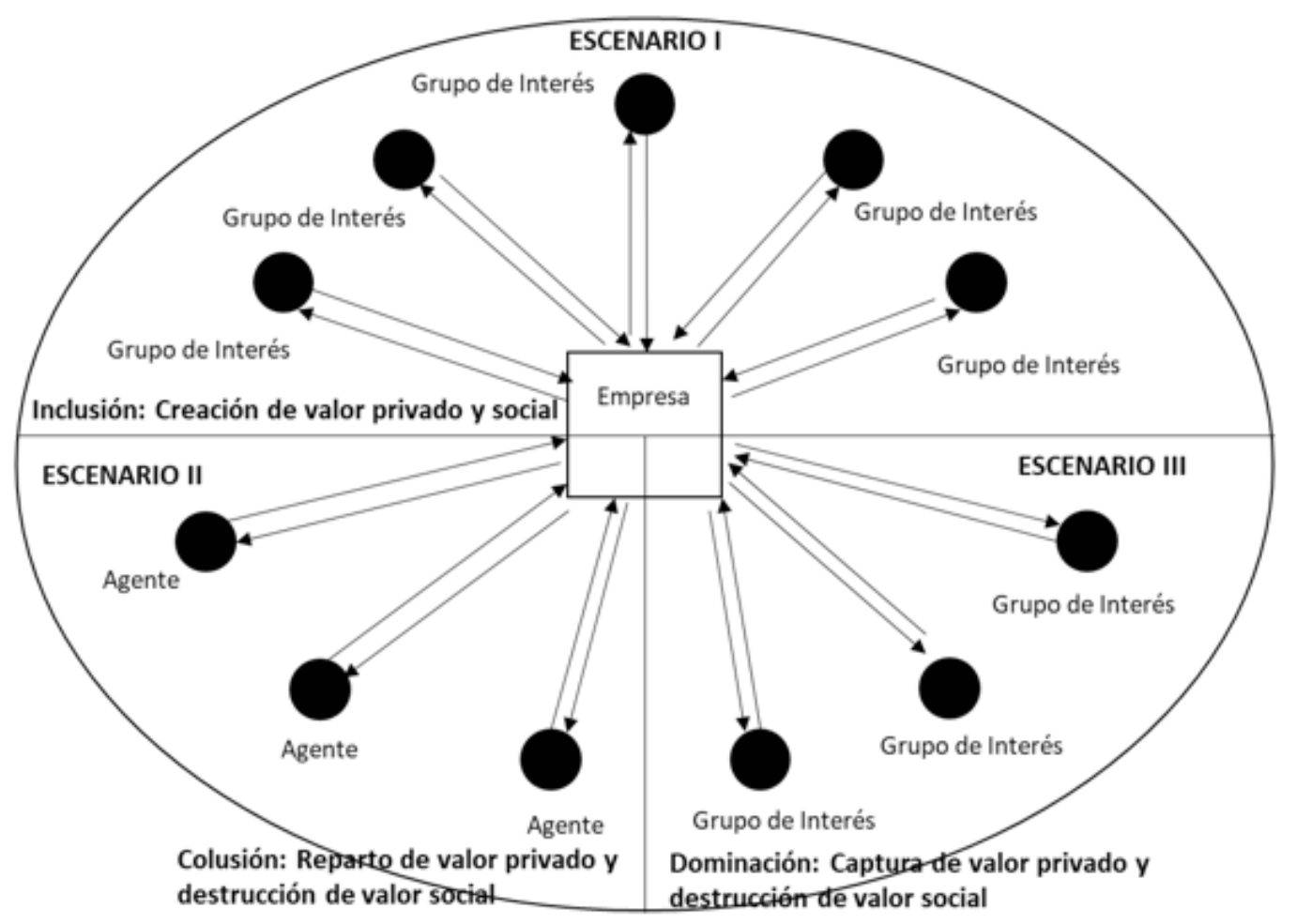

Figura 1. Tipología de vinculación Empresa - Sociedad 


\section{CONCLUSIONES}

La literatura referente a la RSE y la SE no es precisa en la definición de los elementos que la componen. Algunos autores señalan que existe ambigüedad en los conceptos, o subsisten vacíos que no permiten caracterizar los fenómenos con una mayor precisión. Esta situación torna confuso el panorama e impide la instrumentalización de la RSE. Más aún, la RSE carece de elementos propios del contexto latinoamericano, entre otros la corrupción.

Por ello, el artículo repasa distintas definiciones que componen los pilares de la teoría de la RSE y explora concepciones novedosas que pretenden ocupar los vacíos conceptuales reflejados en una deficiente instrumentalización de la RSE. En ese orden de ideas, se han expuesto precisiones respecto al concepto de grupo de interés y legitimidad de los intereses, ISE, y $\mathrm{SE}$, con los que se ha formulado una tipología de vinculación Empresa - Sociedad.

\section{REFERENCIAS BIBLIOGRÁFICAS}

Aidt, T. S. (2011). Corruption and sustainable development. En S. Rose-Ackerman y T. Soreide (Eds.) International Handbook on the Economics of Corruption. Cheltenham, UK: Edward Elgar Publishing

Aras, G. y Crowther, D. (2009). Corporate Sustainability Reporting: A Study in Disingenuity? Journal of Business Ethics, 87(supplement 1), 279-288. https://doi.org/10.1007/s10551-0089806-0

Bansal, P. (2005). The corporate challenges of sustainable development. Academy of Management Executive 16(2), 122-131.

Bansal, P., y DesJardine, M. R. (2014). Business sustainability: It is about time. Strategic Organization, 12(1). 70-78. DOI: 10.1177/1476127013520265

Bowen, H. R. (1953). Social Responsibility of the Businessman, New York, NY: Harper \& Row.

Clegg, S. R. y Hardy, C. (1996). Introduction: Organizations, Organization and Organizing. En S. R. Clegg, C. Hardy y W. R. Nord (Eds) Handbook of Organization Studies. London, UK: Sage.

Donaldson, T., y Preston, L. E. (1995). The Stakeholder Theory of the Corporation: Concepts, Evidence, and Implications. The Academy of Management Review, 20(1), 65. doi:10.2307/258887
Dyllick, T. y Hockerts, K. (2002). Beyond the Business Case for Corporate Sustainability. Business Strategy and the Environment, 11(2), 130-141. DOI: $10.1002 /$ bse.323

Elkington, J. (1994). Towards the Sustainable Corporation: Win-Win-Win Business Strategies for Sustainable Development. California Management Review, 36(2). 90-100. DOI:10.2307/41165746

Freeman, R. E. (1984). Strategic Management A Stakeholder Approach. Boston, MA: Pitman.

Freeman, R. E., Harrison, J. S., Wicks, A. C., Parmar. B. y de Cole, S. (2010). Stakeholder Theory the State of the Art. New York, NY: Cambridge.

Friedman, M. (13 de Setiembre de 1970). The Social Responsibility of Business Is to Increase Its Profits. The New York Times Magazine.

Garriga, E. y Melé, D. (2004). Corporate Social Responsibility Theories: Mapping the Territory. Journal of Business Ethics. 53(1/2), 51-71.

Guillén, J. (2009). ¿Cuál es el origen de la crisis? En G. Alarco (Ed.) Crisis Análisis y perspectivas de la crisis económica mundial desde el Perú. Lima, Perú: Norma.

Hahn, R. (2011). Integrating corporate responsibility and sustainable development. Journal of Global Responsibility, 2(1), 8-22. DOI:10.1108/20412561111128492

Hnyilicza, E. (2009). ¿La teoría económica puede explicar la crisis? En G. Alarco (Ed.) Crisis Análisis y perspectivas de la crisis económica mundial desde el Perú. Lima, Perú: Norma.

Jacobs, G., Van Witteloostuijn, A. y Christe-Zeyse, J. (2013). A theoretical framework of organizational change, Journal of Organizational Change Management, Vol. 26 Issue: 5, pp.772792, https://doi.org/10.1108/JOCM-09-20120137

McElroy, M. W. y Van Engelen, J. M. L. (2012). Corporate Sustainability Management The Art and Science of Managing non-Financial Performance. New York, NY: Earthscan.

Martin, K. D. Johnson, J. L. y Cullen, J. B. (2015). Organizational Change, Normative Control Deinstitutionalization, and Corruption. Business Ethics Quarterly, 19(1), 105-130.

Michael, B. (2004). Explaining organizational change in international development: the role of complexity in anti-corruption work. Journal of International Developemnt, 16(8), 1067-1088. https://doi.org/10.1002/jid.1126 
Montiel, I., \& Delgado-Ceballos, J. (2014). Defining and Measuring Corporate Sustainability. Organization \& Environment, 27(2), 113-139. DOI:10.1177/1086026614526413

Tench, R., Sun, W. y Jones, B. (2012). The challenging concept of corporate social irresponsaibility: An introduction. En R. Tench, W. Sun y B. Jones (Eds.) Corporate Social Irresponsibility: A Challenging Concept. Bradford, UK: Emerald Group Publishing.

United Nations Global Compact. (2018). Pacto Mundial de las Naciones Unidas una llamada a la Acción para Empresas Sostenibles. Descargado de: https://www.pactomundial.org/wp-content/uploads/2018/02/Flyer-New-Strategy-GC-2018_20180126.pdf

Van Marrewijk, M. (2003). Concepts and Definitions of CSR and Corporate Sustainability: Between Agency and Communion. Journal of Business Ethics, 44(2). 95-105. DOI:10.1023/a:1023331212247

Venard, B. (2009). Corruption in emerging countries: A matter of isomorphism. M@n@gement, 12(1), 2-27. http://dx.doi.org/10.3917/ mana.121.0002 
